\title{
HIGH-GRADIENT MEMS ELECTROMAGNETS FOR PARTICLE BEAM MANIPULATION
}

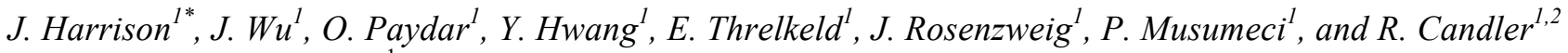 \\ ${ }^{1}$ University of California, Los Angeles, California, USA \\ ${ }^{2}$ California NanoSystems Institute, Los Angeles, California, USA
}

\begin{abstract}
We present the first experimental demonstration of MEMSbased magnetic optics for control of charged particle beams. Combined function (steering and focusing) 4-pole electromagnets with a $600-\mu \mathrm{m}$ bore and $55-\mu \mathrm{m}$ yoke thickness $(686-\mu \mathrm{m}$ magnetic length) have been fabricated with a novel MEMS process. These $3 \mathrm{D}$ solenoidal multi-pole electromagnets have demonstrated hysteresis-free adjustable 2D steering and focusing of a pC-charge 34-keV electron beam with 24-mT field intensity, 220-T/m field gradient, and potential for pulsed operation beyond $100 \mathrm{kHz}$. Simple geometric optimizations promise a 4-fold improvement in both field intensity and gradient without further scaling.
\end{abstract}

\section{INTRODUCTION}

Favorable scaling of magnetic field intensity [1] and mature technology from the magnetic recording industry have made magnetic MEMS inductors, actuators, and relays a common application of microfabrication. These have enabled technologies from miniaturized magnetometers and high-density CMOS passives to high-force actuators and low-insertion-loss RF switches. Applications that use the magnetic field to interact with particles $[2,3]$ are less mature, but can take advantage of the linear scaling of magnetic field gradient with miniaturization of the magnet geometry. One such application is the optical elements used to control charged particle beams: dipole, quadrupole, and higher order fields (Fig. 1). Because the Lorentz force is proportional to particle velocity, forces due to practical magnetic fields dominate Coulomb forces, well before the particles become relativistic (Eq. 1). As a result, strong magnetic optics are necessary in place of electric field optics.
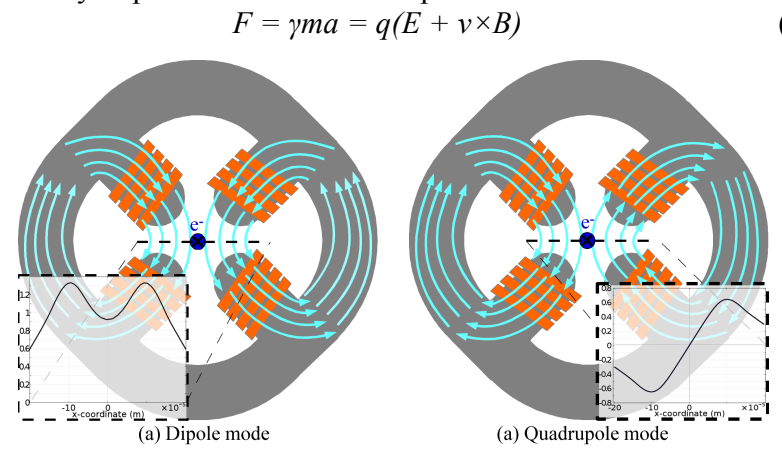

Figure 2. Illustration of a 4-pole MEMS electromagnet operating in dipole field beam steering configuration (left) and quadrupole field beam focusing configuration (right). Inset graphs show magnetic field across the dashed lines.

\section{Multi-pole field scaling}

Modern low-emittance electron and proton beams have transverse dimensions in the $\mu \mathrm{m}$-scale and require intense dipole magnetic fields to steer charged particle beams and strong quadrupole magnetic gradients to maintain focus the beams. As the geometry of a multi-pole electromagnet is scaled down, both steering strength and focusing strength improve. Steering is accomplished with Lorentz force, where the dipole field of an electromagnet can be approximated by $B=\mu n I / 2 r: \mu$ is the electromagnet yoke permeability, $n$ is the number of turns in the electromagnet winding, $I$ is the current in the winding, and $2 r$ is the separation between the electromagnet pole tips. For focusing, the focal length of a magnetic lens can be expressed by $f^{l}=q g l / p: q$ is the particle charge, $g$ is the quadrupole field gradient, $l$ is the magnetic length, and $p$ is the particle momentum. The magnetic length, $l$, is the normalized interaction length between particles and the magnetic field and requires that the electromagnet yoke be a thick enough film $(50+\mu \mathrm{m})$ to change the momentum of the beam. The gradient can be approximated by $g=2 \mu n I / r^{2}$, scaling inversely with the square of the magnet bore.

The reduced inductance of miniaturized electromagnets provides further scaling advantages, allowing the electromagnet to operate pulsed with a reduced duty cycle and at higher frequencies. Background

Many groups have pursued scaling magnetic optics and systems, primarily using machined permanent magnet technology. Tatchin et al. led early efforts with the goal of a miniaturized undulator, using high-precision machining to scale periodic $\mathrm{NdFeB}$ magnets down to 353- $\mu \mathrm{m}$ size [4]; however, device quality was inadequate to realize the promised performance. A more modern effort by Oniku et al. used laser-machined SmCo with $50-\mathrm{mT}_{\text {peak }}$ periodic fields in a $200-\mu \mathrm{m}$ gap [5]. A recent $5 \mathrm{~mm}$ bore $\mathrm{NdFeB}$ Halbach quadrupole now holds the focusing strength record with a field gradient of $560 \mathrm{~T} / \mathrm{m}$ [6], though the machining method resulted in a device size 10x greater than the magnetic optics described here. Our method differs from prior work by using actively powered MEMS electromagnets, enabling powerful and rapidly tunable dipole and quadrupole magnetic fields.

\section{FABRICATION}

The fabrication process for these 4-pole electromagnets was inspired by the UCLA switch [7]. The process was redesigned for multi-pole electromagnets with $55-\mu \mathrm{m}$ thick electromagnet yokes (5x thicker than previous devices) and $2500-\mu \mathrm{m}^{2}$ cross-section windings for high-current electromagnets [8] (Fig. 2).
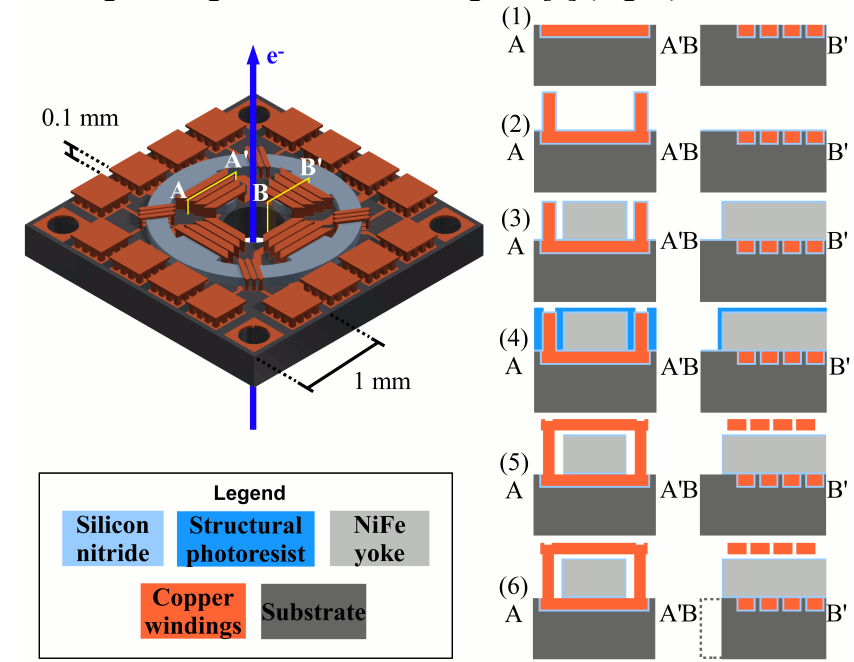

Figure 2: Micro-electromagnet fabrication process. Blue indicates the electron beam path through the device.

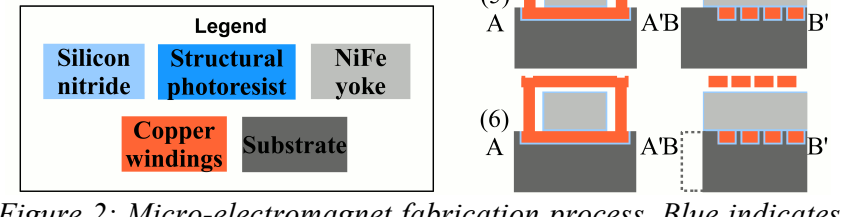

Solid-State Sensors, Actuators and Microsystems Workshop Hilton Head Island, South Carolina, June 8-12, 2014 


\section{(1) Bottom-winding layer}

The pattern for the winding's bottom layer is photolithographically defined on a Si wafer using KMPR 1005 photoresist and an aligner (Karl Süss MA6). Trenches are etched $20-\mu \mathrm{m}$ into the Si through this soft mask using a deep reactive ion etcher (Plasma-Therm SLR-770). The photoresist is stripped in ALEG-380 and 5:1 Piranha. A 500-nm $\mathrm{SiO}_{2}$ film is grown by thermal oxidation (Tystar Mini 3600) to isolate the bottom windings from the wafer. An electroforming seed is deposited on the $\mathrm{SiO}_{2}$ by $\mathrm{RF}$ sputtering (CVC 601). The seed layer consists of 30-nm Ti for adhesion to the substrate and 300-nm Cu to carry the electroplating current. A $25-\mu \mathrm{m} \mathrm{Cu}$ film is electroplated onto the seed layer from a phosphorized $\mathrm{Cu}$ anode in a sulfate based solution (Technic Elevate 6320) through a woven polypropylene filter at $5 \mathrm{~mA} / \mathrm{cm}^{2}$. The wafer is polished to $\mathrm{Si}$ with chemical mechanical polishing, CMP, (Logitech PM5) using 100-nm alumina slurry, yielding the winding's bottom layer inlayed in the substrate. Fig. 3 shows the first layer of a 4-pole electromagnet after CMP.

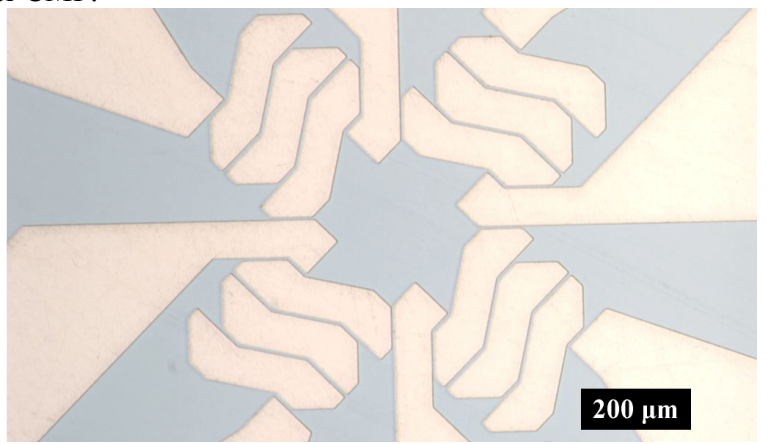

Figure 3: Photograph of $\mathrm{Cu}$ inlaid in a Si wafer, forming the winding's bottom layer of the 4-pole electromagnet.

(2) Winding vias

Another electroforming seed is sputter deposited, with an additional 30-nm layer of $\mathrm{Ti}$ for adhesion between the seed and electroplating mold (i.e., $\mathrm{Ti} / \mathrm{Cu} / \mathrm{Ti}$ layer). A $100-\mu \mathrm{m}$ negative photoresist (KMPR 1025) film is patterned to define the electromagnet winding's interconnect geometry. The seed exposed by the pattern is etched to $\mathrm{Cu}$ in $1 \% \mathrm{HF}$, and $100 \mu \mathrm{m}$ of $\mathrm{Cu}$ is electroplated through the mold. The features are planarized by CMP. The mold is removed by plasma etching with $4: 1 \quad \mathrm{O}_{2}: \mathrm{CF}_{4}$ plasma (STS AOE). The electroplating seed is stripped with a sputter etch and dips in $5 \% \mathrm{C}_{2} \mathrm{H}_{4} \mathrm{O}_{2}$ and $1 \% \mathrm{HF}$. Finally, a $2-\mu \mathrm{m}$ insulating $\mathrm{Si}_{3} \mathrm{~N}_{4}$ film is deposited by plasma enhanced chemical vapor deposition, PECVD, (STS Multiplex CVD) to isolate the windings from the magnetic yoke.

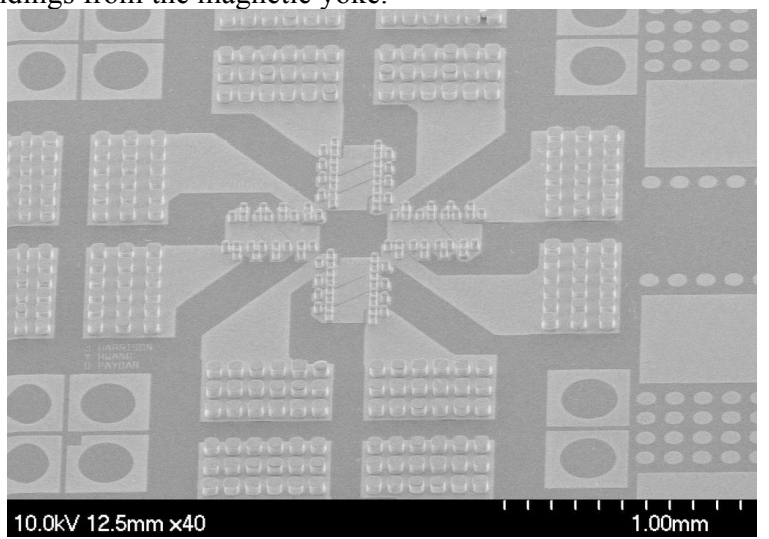

Figure 4: SEM of Cu vias after stripping the electroplating mold.

\section{(3) Magnetic yoke}

An electroforming seed is deposited as described in step 2. A $100-\mu \mathrm{m}$ film of KMPR photoresist is patterned into the geometry of the magnet yoke. Between pouring the photoresist and spinning, the film is de-gassed in a vacuum oven at 30 Torr for $30 \mathrm{sec}$. The exposed $\mathrm{Ti}$ is etched to $\mathrm{Cu}$ in $1 \% \mathrm{HF}$, and a $\mathrm{Ni}_{80} \mathrm{Fe}_{20}$ electromagnet yoke $\left(\mathrm{B}_{\text {sat }}=1.1 \mathrm{~T}, \mu_{\mathrm{r}}=8000\right)$ is plated through the mold using the process detailed by Glickman et al. [8]. Planarization, mold stripping, seed stripping, and isolation proceed as described in step 2. Fig. 5 shows an electromagnet after step 3.

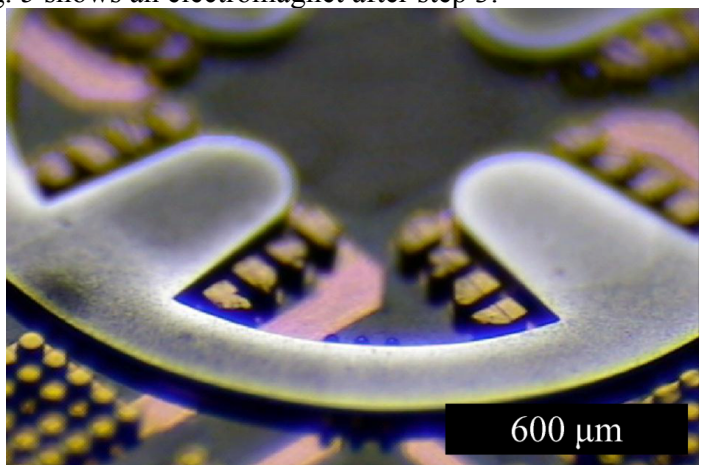

Figure 5: Photograph of NiFe yoke and pole tips after stripping the electroplating mold.

(4) Planarization layer

A $100-\mu \mathrm{m}$ film of photoresist (SU-8 2025) is used to provide a planar surface for defining the top of the coil windings. The photoresist is de-gassed in the same manner as in the previous step, and patterned to expose the winding vias. The photoresist planarized to $10 \mu \mathrm{m}$ above the yoke by CMP between the post exposure bake and development to improve thickness uniformity. The film is annealed in vacuum for 8 hours at $200^{\circ} \mathrm{C}$. Fig. 6 shows multi-pole electromagnets after step 4 .

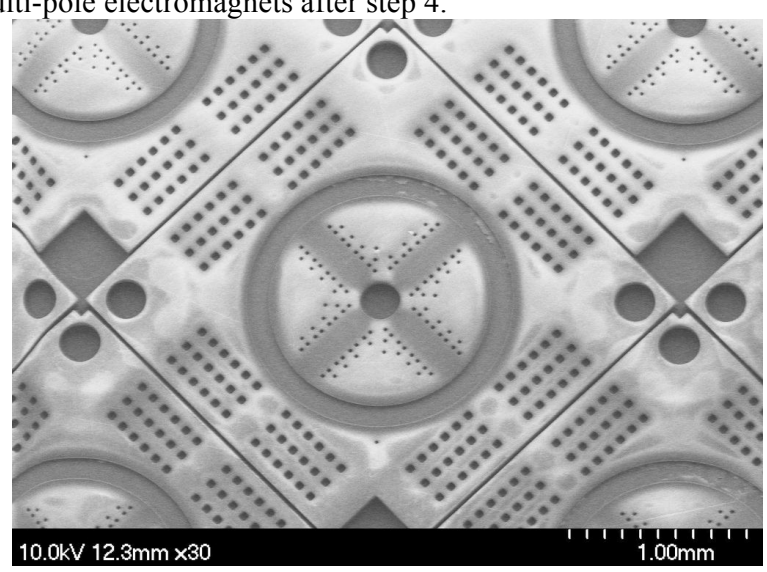

Figure 6: SEM of a 4-pole electromagnet after CMP of the planarization layer.

\section{(5) Top winding layer}

The $\mathrm{Cu}$ in the vias is exposed by etching the $\mathrm{Si}_{3} \mathrm{~N}_{4}$ with $\mathrm{C}_{4} \mathrm{~F}_{8}$ plasma (STS AOE). A seed layer is sputtered on the surface as described in step 2. A $25-\mu \mathrm{m}$ KMPR layer is patterned into the geometry of the top winding layer, and $20-\mu \mathrm{m} \mathrm{Cu}$ is electroplated through the mold. The mold and seed are stripped using the process described in step 2, completing the 4-pole electromagnets. The SU-8 is etched using the mold stripping process described in step 2 to avoid problems from thermal expansion mismatch during operation. Fig. 7 shows an electromagnet after step 5. 


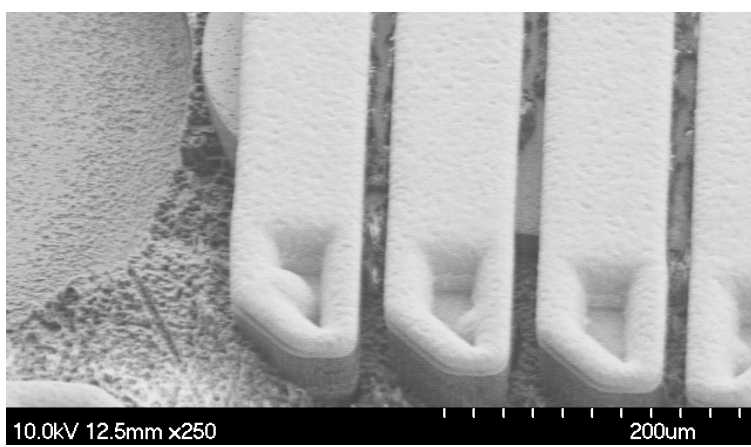

Figure 7: SEM of a 4-pole electromagnet after etching the planarization layer back.

\section{(6) Through wafer etch}

Through-wafer holes are necessary for the particle beam. An etch pattern is defined with KMPR 1005 photoresist on the backside of the wafer and aligned to the front using a contact aligner. Holes and trenches are etched from the back of the substrate to the front using a post-process Bosch etch (Oerlikon FDSE II), defining the electromagnet gap and singulating the devices. Fig. 8 shows an electromagnet after step 6 .

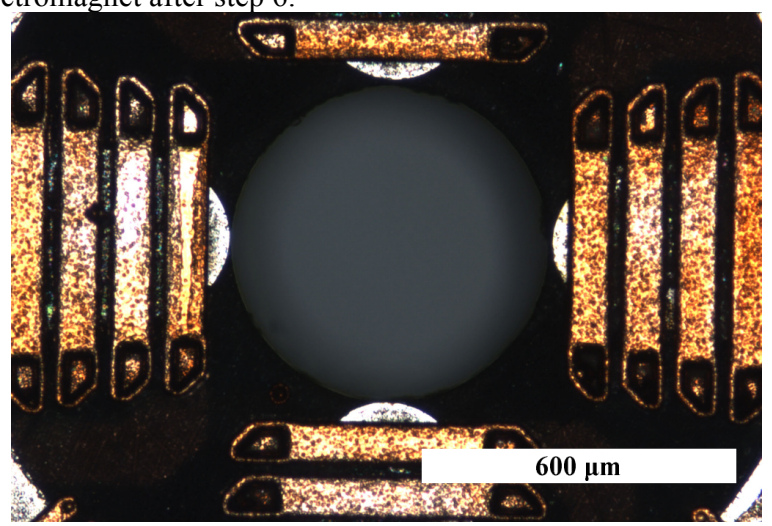

Figure 8: Photograph of a 4-pole electromagnet after throughwafer etching the electron beam path.

(7) Packaging

The 4-pole electromagnet is mounted in a conventionally machined $\mathrm{Cu}$ fixture and wirebonded to an integrated PCB (Rogers Duroid 6002) with $15-\mu \mathrm{m} \quad \mathrm{Al}$ wires. The unpackaged electromagnet die has withstood 70-A pulses on a probe station without failure, but the $15-\mu \mathrm{m} \mathrm{Al}$ wirebonds in the package fail at 5.5 A. Figure 9 shows an electromagnet after step 7.

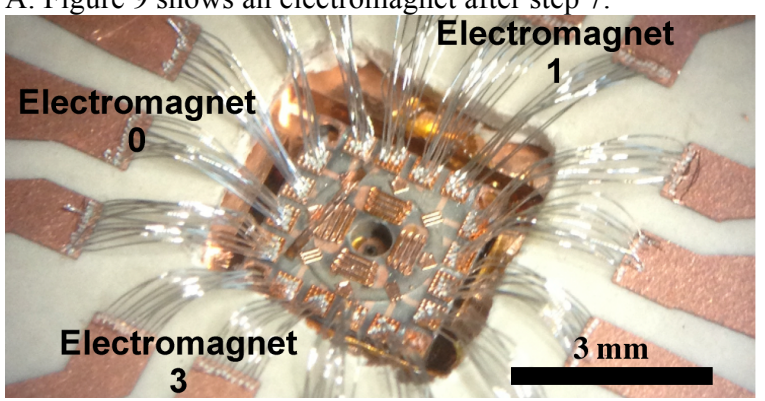

Figure 9: Photograph of a 4-pole electromagnet packaged in a copper beam-testing fixture.

\section{CHARACTERIZATION \& SIMULATION}

Each winding of the electromagnet is measured using an impedance analyzer (Agilent 4294A) with a set of coaxial probes (APT $740 \mathrm{CJ}$ ) in a four-terminal pair configuration. Before packaging, the windings have $58.2 \pm 1.2-\mathrm{m} \Omega$ resistance and
$30.4 \pm 1.9-\mathrm{nH}$ inductance at $100 \mathrm{kHz}$. Fig. 10 shows the electromagnet resistance and inductance vs. frequency after rework and packaging.

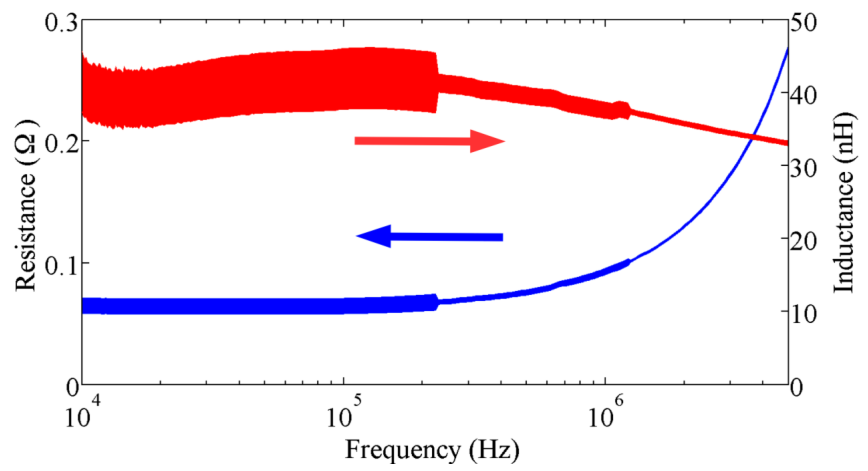

Figure 10: Measured resistance and inductance of the quadrupole shown in Fig. 9. Line thickness denotes measurement precision.

The field produced by $-1.0 \mathrm{~A}$ in each coil of the multi-pole electromagnet was simulated using the finite element method multiphysics software COMSOL. Fig. 10 shows the simulated field profile. The inductance calculated by integrating the stored magnetic energy $\left(E=\int B^{2} / 2 \mu d v\right)$ matched the measured inductance within $6 \%$ before packaging and $25 \%$ after packaging. Postpackaging measurements were through 20 wire bonds reworked with a chlorine plasma etch, potentially explaining the measurement variation.

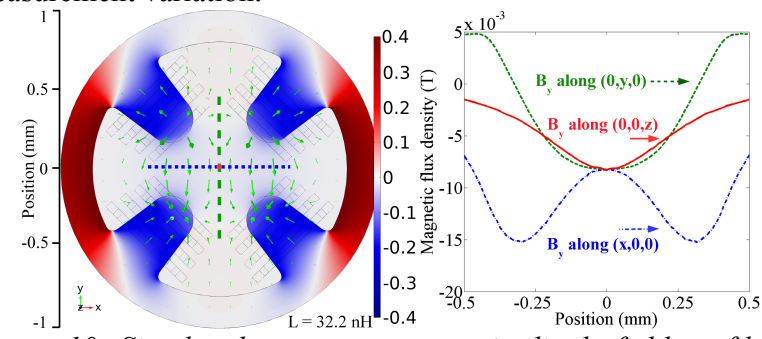

Figure 10: Simulated transverse magnetic dipole field profile for $I=-1.0-A$ drive current along $x$-, $y$-, and $z$-axes. Integration across the model volume yields a 32.2-nH inductance. Dividing the zintegrated field by the peak field yields a 686- $\mu$ m magnetic length, which is the normalized interaction distance of a charged particle.

Simulations show that the electromagnet yoke fully saturates for I $>2.0$-A dipole current, with the poles only $25 \%$ saturated. The yoke width in these devices was limited by a $3-\mathrm{mm}$ die size; however, a $4 \mathrm{x}$ field strength improvement could be realized by widening the yoke without further optimization. Optimized simulations with a $200-\mu \mathrm{m}$ gap rather than $600-\mu \mathrm{m}$ gap exceed $300-\mathrm{mT}$ field and $3-\mathrm{kT} / \mathrm{m}$ field gradient, and $600 \mathrm{mT}$ and $6000 \mathrm{~T} / \mathrm{m}$ with a higher-saturation yoke $\left(\mathrm{B}_{\mathrm{sat}}=2.1 \mathrm{~T}\right.$ for CoNiFe $)$.

\section{EXPERIMENT}

The MEMS electromagnet was mounted in the path of an electron beam following a translatable iris and powered in dipole or quadrupole configuration to demonstrate beam steering and focusing. By varying the electrical current in each coil, the electron beam was steered across an imaging system. The magnetic field could then be calculated from the centroid on the image using Lorentz force and the magnetic length from FEM simulation. By translating the beam iris with the electromagnet field constant, the spatial distribution of the magnetic field was mapped.

The experiment uses an electron beam generated by photoelectric effect with a UV laser and $\mathrm{Cu}$ cathode accelerated with an electric field. A solenoid electromagnet adjusts beam focus 
exiting the electron gun, and a set of steering electromagnets adjusts position and angle. After a drift length, a chamber houses the MEMS electromagnet, and after another drift length, an imaging system composed of a Z-stack micro-channel plate (MCP) intensifier, phosphor screen, and cooled CCD camera (Hamamatsu Flash 2.8) images the beam position and shape. The MEMS electromagnet is mounted behind a pair of micrometer-mounted orthogonal $20-\mu \mathrm{m}$ slits (Thorlabs S20R) that have been stripped of anodizing and iron oxide coatings with $10 \% \mathrm{HCl}$ and $10 \% \mathrm{NaOH}$.

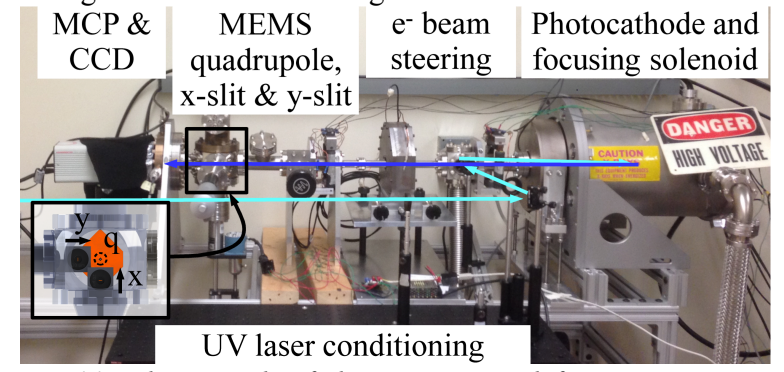

Figure 11. Photograph of the steering and focusing experiment. Cyan is the laser used to extract electrons from the photocathode and blue is the electron path. The beam is imaged using a microchannel plate intensifier and cooled CCD. The inset shows the inside of the experiment chamber, with the MEMS quadrupole ' $q$ ' entering from above, and the horizontal and vertical slits that form the beam iris, ' $y$ ' and ' $x$ ', entering from the left and below.

All beam measurements were calibrated by subtracting an image of the MCP with the electron beam off. The electron beam was slightly under-focused, $34 \mathrm{keV}$, sub-pC, and pulsed at $1 \mathrm{kHz}$. Iris centering was accomplished by switching the electromagnet on and off in quadrupole configuration and translating the iris until there was no movement of the beam on MCP. Each measurement consisted of 25 images taken with $100 \mathrm{~ms}$ exposure time.

Electromagnet current was stepped from $-1.5 \mathrm{~A}$ to $+1.5 \mathrm{~A}$, and back to $-1.5 \mathrm{~A}$ in $\mathrm{x}$ - and $\mathrm{y}$-deflecting configurations. Fig. 8 shows the beam centroid deflection. The measurements demonstrated less than $0.7 \%$ electromagnet hysteresis throughout all electromagnets, a value within the experimental error.

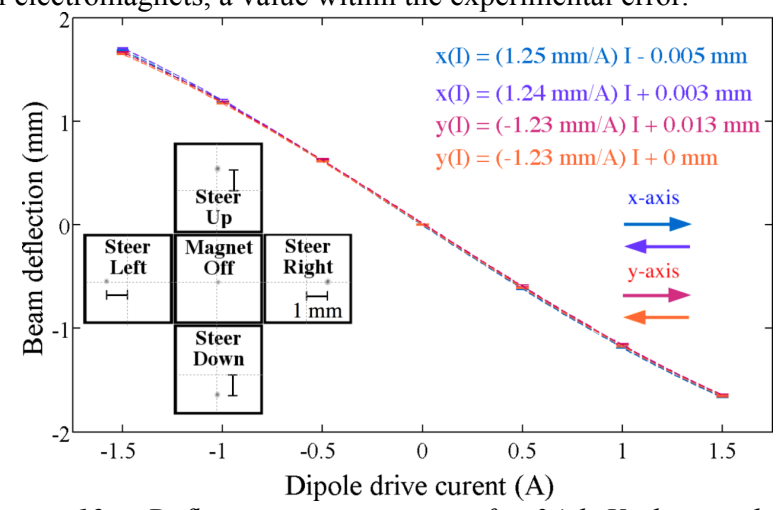

Figure 12. . Deflection measurement of a $34 \mathrm{keV}$ electron beam using the electromagnet in Figure 3 powered in dipole configuration. Measured hysteresis is within the experiment error.

Electromagnet current was fixed at $-1 \mathrm{~A}$ in an $\mathrm{x}$-focusing quadrupole configuration while the iris slit was translated across the $\mathrm{x}$-axis of the electromagnet bore to map the field distribution. The beam centroid was measured with the electromagnet on and off to provide a differential measurement of the beam translation. Fig. 13 shows the measured field across the electromagnet bore. The inset image in Fig. 13 shows the normalized intensity profile of the electron beam with different focusing currents.

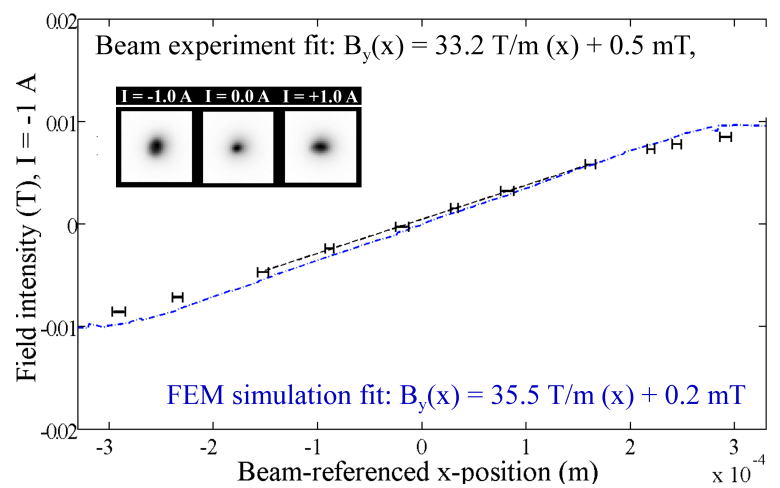

Figure 13. Field measurement and simulation of the electromagnet powered in quadrupole configuration. The inset images show normalized beam shape under $x$ - and $y$-focusing currents.

\section{CONCLUSION}

We have fabricated 4-pole electromagnets with $600-\mu \mathrm{m}$ bore and $55-\mu \mathrm{m}$ thickness using a novel process. Hysteresis-free 24-mT fields and linear $33-\mathrm{T} / \mathrm{m}$ field gradients have been measured with a 34-keV electron beam, marking the first demonstration of $3 \mathrm{D}$ micro-electromagnets for particle beam manipulation. At saturating currents, $220 \mathrm{~T} / \mathrm{m}$ is produced, and gradients exceeding $3 \mathrm{kT} / \mathrm{m}$ are possible by scaling the design to a $200-\mu \mathrm{m}$ gap.

\section{ACKNOWLEDGEMENT}

The authors would like to thank the UCLA Nanolab and ISNC for use of their fabrication facilities and gratefully acknowledge support from DARPA \#N66001-12-1-4209 and NSF \#1350034.

\section{REFERENCES}

[1] J.W. Judy, "Microelectromechanical systems (MEMS): fabrication, design and applications," Smart Mater. Struct., 10, 6, (2001).

[2] P. Tseng, J.W. Judy, D. Di Carlo, "Magnetic nanoparticlemediated massively parallel mechanical modulation of singlecell behavior," Nat. Methods, 9, (2012).

[3] N. Pamme and C. Wilhelm, "Continuous sorting of magnetic cells via on-chip free-flow magnetophoresis", Lab Chip, 6 (2006).

[4] R. Tatchyn and P. Csonka, "Attainment of submillimeter periods and a $0.3 \mathrm{~T}$ peak field in a novel micropole undulator device", App. Phys. Lett., 50, 377 (1987)

[5] O.D. Oniku, B. Peterson, W.C. Patterson, A. Garraud, F. Herrault, M.G. Allen, and D.P. Arnold, "Progress toward mini and micro magnetic undulators", Proceedings of the 2013 workshop on Physics and Applications of High Brightness Beams, San Juan, PR, 3/25-28/13, ICFA, Batavia (in print).

[6] J.K. Lim P. Frigola, G. Travish, J.B. Rosenzweig, S.G. Anderson, W.J. Brown, J.S. Jacob, C.L. Robbins, and A.M. Tremaine, "Adjustable, short focal length permanent-magnet quadrupole based electron beam final focus system", Phys. Rev. STAB, 8, 072401 (2005).

[7] M. Glickman, P. Tseng, J. Harrison, T. Niblock, I.B. Goldberg, and J. W. Judy, "High-Performance LateralActuating Magnetic MEMS Switch", JMM, 20, 842 (2011)

[8] J. Harrison, O. Paydar, Y. Hwang, J. Wu, E. Threlkeld, P. Musueci, andn R. Candler, "Fabrication process for thick-film micromachined multi-pole electromagnets", JMEMS(in print)

\section{CONTACT}

*J.Harrison, tel: +1-623-695-0417; jere.harrison@ucla.edu 